\title{
Effects of Couple Stress on the Growth Rate of Rayleigh- Taylor Instability at the Interface in a Finite Thickness Couple Stress Fluid
}

\author{
N. Rudraiah ${ }^{1}$ and G. Chandrashekara ${ }^{2}$ \\ ${ }^{1}$ UGC-Center of Advanced Studies in Fluid Mechanics, Department of Mathematics, Bangalore University, \\ Central College Campus, Bangalore-560001 \\ ${ }^{2}$ National Research Institute for Applied Mathematics (NRIAM), \#429/G, 7th cross, 7th Block (West), \\ Jayanagar, Bangalore-560070 \\ Email: ${ }^{1}$ rudraiahn@hotmail.com, ${ }^{2}$ chandrashekarg.4@gmail.com
}

(Received November 15, 2008; accepted July 4, 2009)

\begin{abstract}
In this paper the effects of couple stress fluid on the control of Rayleigh-Taylor instability at the interface between a dense fluid accelerated by a lighter fluid is studied. A simple theory, based on fully developed approximations, is used to derive the growth rate of Rayleigh-Taylor instability. The cutoff and maximum wave numbers and the corresponding maximum frequency are obtained. It is shown that the effect of couple stress parameter reduces the growth rate considerably compare to the classical growth rate in the absence of couple-stress and hence favorable to control the growth rate of surface instabilities in many practical problems.
\end{abstract}

Keywords: Micropolar Fluid Theory- Complex Fluid- Coronary Artery Diseases- Synovial Joints-Inertial Fusion Energy.

\section{NomenClature}

$\begin{array}{ll}B & \text { Bond number } \\ e_{i j} & \text { strain tensor } \\ g & \text { acceleration due to gravity } \\ \mathrm{G}_{\mathrm{s}} & \text { angular velocity } \\ G_{m} & \text { ratio of growth rates } \\ h & \text { length scale } \\ l & \text { wave number } \\ L_{i} & \text { angular momentum } \\ M_{i j} & \text { body moment } \\ n & \text { dispersion relation (growth rate) } \\ p_{i} & \text { linear momentum } \\ p & \text { pressure } \\ q_{i} & \text { the velocity } \\ r_{i} & \text { position vector } \\ S & \text { Strouhal number }\end{array}$

$\begin{array}{ll}T & \text { time scale } \\ U & \text { Characteristic velocity } \\ \tau_{i j}^{\prime} & \text { angular stress tensor } \\ \tau_{i j} & \text { shear stress tensor } \\ v & \text { kinematic viscosity } \\ \tau_{j k} & \text { stress tensor } \\ \Omega_{i} & \text { vorticity tensor } \\ \lambda & \text { couple stress coefficient } \\ \rho & \text { density of the fluid } \\ \gamma & \text { surface tension } \\ \mu & \text { coefficient of viscosity } \\ \sigma & \text { couple stress parameter } \\ \mu^{\prime}, & \mu \text { dimensions of viscosity } \\ \varepsilon_{i j k} & \text { the Levi - Civita symbol } \\ \eta & \text { elevation of the interface }\end{array}$




\section{INTRODUCTION}

The interfacial instabilities continue to be the frontier area of research in understanding, control and exploitation of micro fluidic devices. The complex fluid flows through micro channels have been developing rapidly because of their applications in biosciences, physical sciences, in the effective design of artificial organs in biomedical engineering, inertial fusion energy (IFE), solidification process in material science, heat transfer across barriers, friction between surfaces and their mitigation, in adhesion and failure of polymers and so on. For efficient design of artificial organs in human body like synovial joints, endothelium in coronary artery diseases (see $\mathrm{Ng}$ et al. 2005), efficient extraction of IFE (see Rudraiah, 2003; Rudraiah and Milan, 2007) and so on, it is essential to control the growth rate of surface instabilities. The following three types of surface instabilities are given considerable interest in the literature (see Chandrasekhar, 1961; Rudraiah, 2003),

1. Rayleigh-Taylor Instability [RTI]

2. Kelvin-Helmholtz Instability [KHI]

3. Richtmayer-Meshkov Instability [RMI].

RTI occurs at an interface between highly dense and less dense fluids when the latter are at high pressure. KHI occurs at an interface subject to shear. RMI is regarded as a special case of RTI and it corresponds to the case of shock-accelerated interface. These instabilities are extensively investigated (see Rudraiah and $\mathrm{Ng}, 2004$; Bhatia, 1974) in ordinary Newtonian and electrically conducting fluids. However, the Biomechanical and industrial problems mentioned above involve non-Newtonian fluids (see Sharma and Sharma, 1978 and Rudraiah et al. 2000). Sharma and Sharma (1978) have studied RTI using Oldroyed's viscoelastic model, where as Rudraiah et al. (2000) have studied RTI using power law model. In biomedical problems for example in synovial joints the synovial fluid is bounded by porous nature of cartilages and in coronary arterial diseases (CAD) the blood in arteries is bounded by porous nature of endothelium the boundaries of the arteries. When joints degenerate the irregular shape of the interface between cartilages and synovial fluid deforms and produce surface instabilities. In CAD the cholesterol and other fat substances will accumulate on the endothelium and form the plaques (i.e. the growth) on the endothelium. If the plaques reach a critical stage it will block the flow of blood in arteries and a cardiologist resort to by-pass or other devices like angiograms and soon. In these days, a high intensity laser is used to melt the plaque. Although the laser melts the plaques, it erodes the endothelium causing surface instability of the RTI type. To prevent such side effects there is a need to understand the growth rate of surface instability of the RTI type.

During the degenerative changes either in synovial joints or in CAD the fluid deforms and produces a spin field due to their micro rotation. The experiments of Christopherson and Dowson (1959), using the solid spheroid model support this. This spin field sets up an anti-symmetric stress known as "Couple stress". Rudraiah et al. (1991) have shown, using the generalized dispersion model of Gill and Sankarasubramanian (1970), the effect of couple stress is to facilitate the loss of hemoglobin, a disease known as haemolysis in the blood. To understand the control of such situation there is a need to study the surface instability of the type RTI. To our knowledge RTI in the design of the above artificial organs in biomedical engineering using a couple stress fluid, a particular case of micro polar fluid, has not been given much attention and the study of it is the main objective of this paper.

To achieve this objective, the plan of this paper is as follows. In section 2, on formulation of the problem, the physical configuration, the basic equations and the required boundary conditions are given. In section 2.1 the solution of the differential equation is obtained by using the lubrication and Stokes approximations. The dispersion relation is derived in section 3 with and without the couple stress effect. To know the nature of the growth rate, the ratio of maximum growth rate to classical growth rate is computed for different values of couple stress parameters and the results are tabulated in Table 1 . The growth rate $n$ is computed for different values of $\sigma, B$ and $l$ and the results are represented graphically in Figs. 2 and 3. Important conclusions are drawn in the final section.

\section{Formulation of the Problem}

The physical configuration, shown in Fig. 1, consists of a thin film of an unperturbed thickness $2 \mathrm{~h}$ filled with an incompressible viscous couple stress lighter fluid of constant density $\rho_{1}$ called Region 1 bounded below by a rigid surface and above by a dense incompressible heavy couple stress fluid of density $\rho_{2}$ called the Region 2, with the interface at $y=h$.

The rheological properties of physiological fluids like synovial fluid in synovial joints, blood flow in arteries reveal that viscosity varies nonlinearly with concentration exhibiting either shear thinning or shear thickening behaviour. This is one of the non-Newtonian fluid flow properties. Most of the existing literature (see Fung, 1981; Rudraiah, 1998) on this is silent about micro motions, micro rotation and deformations. These are taken into account in this paper using a couple stress fluid as a particular case of micropolar fluid theory developed by Eringen (1966) as described by Rudraiah et al. (1998). The basic equations for this fluid are

The conservation of mass for an incompressible fluid

$$
\frac{\partial q_{i}}{\partial x_{i}}=0
$$

The conservation of linear momentum:

$$
\rho \frac{D q_{i}}{D t}=\frac{\partial \tau_{i j}}{\partial x_{j}}+\pi_{i}+\rho f_{i}
$$

Conservation of angular momentum: 
Angular momentum is usually defined as the moment of the linear moment. If $r_{i}$ is the position vector of the particles and

$$
p_{i}=\rho q_{i}
$$

is the linear momentum, then the angular momentum,

$$
\begin{aligned}
& L_{i}, \text { is } \\
& L_{i}=\varepsilon_{i j k} r_{i} p_{k}
\end{aligned}
$$

where $\varepsilon_{i j k}$ is the Levi - Civita symbol defined as

$$
\varepsilon_{i j k}=\left\{\begin{array}{l}
1 \quad \text { if } i, j, k \text { take values in cyclic order } \\
-1 \text { if } i, j, k \text { take values in acyclic order } \\
0 \text { if two or all of } i, j, k \text { take the same value }
\end{array}\right.
$$

The conservation of angular momentum, neglecting the body couple and contact couples (see Rudraiah, 1998) can be obtained, by taking the cross product of $r_{i}$ with Eq. (2), and using Eq. (3), in the form

$\varepsilon_{i j k} r_{i} \frac{\partial p_{k}}{\partial t}+\varepsilon_{i j k} r_{i} \frac{\partial q_{j}}{\partial x_{k}} p_{k}=\varepsilon_{i j k} r_{i} \frac{\partial \tau_{j k}}{\partial x_{k}}+\varepsilon_{i j k} \rho r_{i} f_{k}$

In Eqs. (1) to (5), $q_{i}(\mathrm{i}=1,2,3)$ are the velocity components, $\tau_{j k}$ is the stress tensor and $\rho f_{k}$ is the body force.

We note that the limitations encountered in the continuum theory are the lack of taking into account the micro rotation of hyaluronic acid (HA) molecules present in synovial fluid. In that case, the intrinsic motions of the micro elements must be taken into account because the microelement motions and deformations play a significant role in deriving the required constitutive equations. In such situations, the Eringen's (1966) "micro polar fluid" theory is useful and he showed that the couple stress theory results as a special case of micro polar fluid theory when the micro rotation vector is constrained equal to the fluid bulk vorticity through out the flow field, and the deformation of the fluid microelements is considered to be very small. Then, the constitutive equations for couple stress fluid, following Stokes (1968) as in Rudraiah (1998), are

$\tau_{i j}=\left(-p+\mu^{\prime} e_{k k}\right) \delta_{i j}+2 \mu e_{i j}$,

(the shear stress tensor)

$\tau_{i j}^{\prime}=-2 \lambda \Omega_{i j, k k}-\frac{\rho}{2} \varepsilon_{i j s} G_{s}$,

(the angular stress tensor)

$$
M_{i j}=4 \lambda \Omega_{j, i}+4 \lambda^{\prime} \Omega_{i, j}
$$

$e_{i j}=\frac{1}{2}\left(\frac{\partial q_{i}}{\partial x_{j}}+\frac{\partial q_{j}}{\partial x_{i}}\right)$ is the strain tensor

$$
\Omega_{i}=\varepsilon_{i j k} q_{k, j}=\frac{1}{2}\left(\frac{\partial q_{i}}{\partial x_{j}}-\frac{\partial q_{j}}{\partial x_{i}}\right)
$$

(is the vorticity tensor)

$\mathrm{G}_{\mathrm{s}}$ is the angular velocity vector and the $M_{i j}$ is the body moment.

Here the dimensions of $\mu^{\prime}$ and $\mu$ are those of viscosity and $\lambda$ and $\lambda^{\prime}$ are those of momentum. The ratio $\frac{\lambda}{\mu}$ has the dimensions of length squared.

For an incompressible fluid, when the body moments are absent, the basic equations of motion for couple stress fluid, using Eqs. (5) to (7), following Rudraiah (1998), are

$\rho\left(\frac{\partial q_{i}}{\partial t}+q_{i} \frac{\partial q_{i}}{\partial x_{j}}\right)=-\frac{\partial p}{\partial x_{i}}+\mu \frac{\partial^{2} q_{i}}{\partial x_{i}^{2}}-\lambda \frac{\partial^{4} q_{i}}{\partial x_{i}^{4}}$

These equations have to be solved satisfying the no-slip and couple stress boundary conditions given in the subsequent section.

\subsection{Solution of the Problem}

To study the problem of Rayleigh Taylor instability posed in this paper, we consider the fully developed, steady and unidirectional flow of lighter couple stress fluid in Region 1 accelerating the heavy dense couple stress fluid in Region 2. To obtain the required equations, we use the following combined lubrication and stokes approximations:

1. The thickness $2 h$ of the lighter fluid is much smaller than the thickness $H$ of the dense fluid above the interface, that is

$$
2 h<<H
$$

2. The Strouhal number, $S$, which is the measure of the local acceleration to the inertial acceleration, is

$$
\mathbf{S}=\frac{\mathbf{L}}{\mathbf{U T}}<<\mathbf{1}
$$

Here $U=\frac{v}{L}$ is the characteristic velocity, $v=\frac{\mu}{\rho}$ the kinematic viscosity, $L=\sqrt{\frac{\gamma}{\delta}} \quad$ the characteristic length, $\gamma$ the surface tension, $\delta=g\left(\rho_{1}-\rho_{2}\right)$ and $T=\frac{\mu \gamma}{h^{3} \delta^{2}} \quad$ is the characteristic time scale. The assumption (13) enabled us to neglect the local acceleration term $\frac{\partial q_{i}}{\partial t}$ in Eq. (11). 
3. We consider high viscous couple stress fluid so that inertial acceleration term in Eq. (11), can be neglected comparing with viscous term.

4. The interface elevation, $\boldsymbol{\eta}$, is assumed to be small compared with film thickness $2 h$ that is

$$
\frac{\eta}{h}<<1
$$

These assumptions enabled us to use the creeping flow approximation which allows us to neglect certain terms in the perturbation equation to obtain linear equations for the interface elevation.

Under these assumptions, for a two dimensional flow the basic Eqs. (11) and (1), respectively reduce to the form

$0=-\frac{\partial p}{\partial x}+\mu_{f} \frac{\partial^{2} u}{\partial y^{2}}-\lambda \frac{\partial^{4} u}{\partial y^{4}}$

$0=-\frac{\partial p}{\partial y}$

$0=\frac{\partial u}{\partial x}+\frac{\partial v}{\partial y}$

These equations are solved using the following boundary and surface conditions:

The no-slip boundary condition at the rigid surface

$\mathrm{u}, \mathrm{v}=0 \quad$ at $\mathrm{y}=-\mathrm{h}$

The interfacial boundary condition

$$
\frac{\partial u}{\partial y}=0 \text { at } y=h
$$

The couple stress boundary condition

$$
\frac{\partial^{2} u}{\partial y^{2}}=0 \text { at } y= \pm h
$$

The dynamic surface condition at the interface

$$
p=-\delta \eta-\gamma \frac{\partial^{2} \eta}{\partial x^{2}} \text { at } y=h
$$

The kinematic surface condition at the interface

$$
v=\frac{\partial \eta}{\partial t}+u \frac{\partial \eta}{\partial x} \text { at } \mathrm{y}=\mathrm{h}
$$

For the linear case the above Eq. (21) reduces to the form

$v=\frac{\partial \eta}{\partial t}$ at $y=h$
Making Eqs. (14) to (22), dimensionless, using the scales, h for length, $\delta h$ for pressure, $\left(\frac{\delta h^{2}}{\mu_{f}}\right)$ for velocity and $\left(\frac{\mu_{f}}{\delta h}\right)$ for time, we get

$\frac{\partial^{4} u}{\partial y^{4}}-\sigma^{2} \frac{\partial^{2} u}{\partial y^{2}}=-\sigma^{2} \frac{\partial p}{\partial x}$

where $\sigma^{2}=\frac{\mu_{f} h^{2}}{\lambda}$ is the couple stress parameter.

Solution of Eq. (23), using the dimensionless form of boundary conditions (17) to (22), is

$u=\left[\frac{1}{\sigma^{2}}\left(1-\frac{\cosh \sigma y}{\cosh \sigma}\right)-\left(\frac{1-y^{2}}{2}\right)-(1+y)\left(1-\frac{\tanh \sigma}{\sigma}\right)\right] \frac{\partial p}{\partial x}$

\subsection{Dispersion Relation}

Integrating Eq. (16), over the limits -1 to 1 and using the condition (17), we get

$v(1)=-\int_{-1}^{1} \frac{\partial u}{\partial x} d y$

Then using Eq. (24), and integrating, we get

$v(1)=\eta_{0} e^{i l x+n t}\left[\frac{2}{3}+\frac{2\left(\sigma^{2}-1\right)}{\sigma^{2}}\left(1-\frac{\tanh \sigma}{\sigma}\right)\right] \frac{\partial^{2} p}{\partial x^{2}}$

Making Eq. (22), dimensionless using the scales defined above and assuming the normal mode solution of the form $\eta=\eta_{0} e^{i l x+n t}$, we get

$v(1)=n \eta_{0} e^{i l x+n t}$

From Eq. (26), with normal mode solution and using Eq. (27), we get the dispersion relation in the form

$\mathrm{n}=\left\{\frac{1}{3}-\frac{6\left(1-\sigma^{2}\right)\left(1-\frac{\tanh \sigma}{\sigma}\right)-\sigma^{2}}{3 \sigma^{2}}\right\} l^{2}\left(1-\frac{l^{2}}{B}\right)$

where $B=\frac{\delta h^{2}}{\gamma}$ is the Bond number and $n$ is the growth rate in the presence of couple stress effect.

In the absence of couple stress, that is $\lambda \rightarrow 0$ (that is $\sigma^{2} \rightarrow \infty$ ), the dispersion relation Eq. (28), reduces 
$n_{b}=\frac{l^{2}}{3}\left(1-\frac{l^{2}}{B}\right)$

which is the expression given by Babchin et al. (1983). Then Eq. (28), can be written as

$n=n_{b}-\beta l v_{a}$

where $n$ is the growth rate of the interface, $l$ is the wave number, $\beta=\frac{1}{\sigma^{2}}$

$$
v_{a}=\left[2\left(1-\sigma^{2}\right)\left(1-\frac{\tanh \sigma}{\sigma}\right)-\frac{1}{3}\right] l\left(1-\frac{l^{2}}{B}\right)
$$

the velocity across the ablation front, $\sigma$ the couple stress parameter and $n_{b}$ is given in Eq. (29).

\section{Discussion AND CONCLUSIONS}

The linear RTI of an incompressible viscous couple stress fluid in a thin film shell bounded below by a rigid surface and above by a dense incompressible heavy couple stress fluid of high density is studied using normal mode technique. The dispersion relation given by Eq. (30) is derived.

By setting $n=0$ in the dispersion relation (28), we get the critical cut-off wave number as

$l_{c t}=\sqrt{B}$

The maximum wave number $l_{m}$, obtained from Eq. (28), by setting $\frac{\partial n}{\partial l}=0$, is

$l_{m}=\frac{l_{c t}}{\sqrt{2}}$

We note that relations (31) and (32) are true even in the absence of couple stress effect and for convenience we call them as classical results.

The maximum growth rate, obtained from Eq. (28), using Eq. (32), is

$n_{m}=\frac{B}{2}\left(\frac{\sigma^{2}-1}{\sigma^{2}}\right)\left(1-\frac{\tanh \sigma}{\sigma}\right)$

Similarly, from Eq. (29), using Eq. (32), we get the maximum classical growth rate as

$n_{m_{b}}=\frac{B}{2}$

The ratio of the growth rates, $G_{m}$, obtained from Eqs. (33) and (34), is
$G_{m}=\frac{n_{m}}{n_{m_{b}}}=\left(1-\frac{1}{\sigma^{2}}\right)\left(1-\frac{\tanh \sigma}{\sigma}\right)$

This $G_{m}$ is computed for different values of couple stress parameter $\sigma$ and the results are tabulated in Table 1. The percentage of reduction and values of $G_{m}$ are compared with those give by Takabe et al. (1985), Rudraiah (2003) and Rudraiah and Milan (2007) and we conclude that the reduction of the growth rate by couple stress fluid is more significant that those obtained by these authors. In particular, we note that the maximum reduction of growth rate is $99 \%$ for $\sigma=1.01$, compared to $45 \%$ reduction predicted by Takabe et al. (1985). Thus, the presence of couple stress is very effective in reducing the asymmetry in IFE target caused by fusing Deuterium and Tritium (D-T) and hence facilitates to increase the efficiency of extraction of IFE by fusing D-T. These results are also useful in the effective design of artificial organs. For example, in removing plaques (that is growth) formed on the endothelium, the walls of the coronary artery and thus helps in controlling the coronary artery diseases.

The frequency $n$ given by Eq. (28) is computed for different values of $\sigma$ and B and the results are graphically represented in Figs. 2 and 3. Figure 2 is the graph of $n$ versus $l$ for different values of $\sigma$. From this Fig. 2, it is clear that a decrease in $\sigma$ (that is increase in $\lambda$ ) decreases the growth rate and hence makes the RTI more stable. Figure 3 is the graph of $n$

versus $l$ for different values of Bond number B, which is the reciprocal of surface tension. The increase in $\mathrm{B}$ implies decrease in surface tension. In this Fig. 3 the frequency, $n$ increases with an increase in B implying decrease in surface tension. That is decrease in surface tension make the system more stable as expected on physical grounds.

\section{ACKNOWLEDGEMENTS}

This work is supported by ISRO under the research projects no. ISRO/RES/2/338/2007-08 and ISRO/RES/2/335/2007-08. ISRO's financial support to carry this research is gratefully acknowledged. One of us (G.Chandrashekara) gratefully acknowledges ISRO for providing JRF under the above project

\section{REFERENCES}

Babchin, A.J, A.L. Frenkel and B.G. Levich (1983). Non-linear saturation of Rayleigh-Taylor instability in a thin film. Phy. Fluids 26, 3159.

Bhatia, P.K. (1974). Rayleigh-Taylor instability of two viscous superposed conducting fluids. Nuovo Cim. 19B, 161.

Chandrasekhar, S. (1961). Hydrodynamic and hydromagnetic stability. Dover Publication, New York. 
Christperson, D.C. and Dawson (1959). An example of minimum energy dissipation in viscous flows. Proc. Ray. Soc. A251, 550.

Eringen, A.C. (1966). Theory of Micropolar Fluids. J. Math. Mech. 16, 1.

Fung, Y.C. (1981). Biomechanics. Springer and Verlag, New York.

Gill and Sankarasubramanian (1970). Exact analysis of unsteady convective diffusion. Proc. Ray. Soc. A316, London, 347.

Ng, C.O., N. Rudraiah, C. Nagraj and H.N. Nagraj (2005). Electrohydrodynamic dispersion of macromolecular components in nanostructure biological bearing. J. of Energy, Heat and Mass Transfer 27, 39.

Rudraiah, N. and C.O. Ng (2004). A model for manufacture of nanostructure of nano size smart material free from impurities. Current Sci. 86, 8.

Rudraiah, N. (2003). Effect of porous lining on reducing the growth rate of Rayleigh-Taylor instability in the inertial fusion energy target. Fusion Sci., and tech.43, 307-311.

Rudraiah, N., P. Sridharan and T. Desai (2003). Laser driven ablative surface instability in the inertial fusion energy. Int. J. Applied Mech. and Engg. 8(4), 665-676.

Rudraiah, N., S. Prema and S. Bhargava (2000). Rayleigh-Taylor instability in a finite thickness of a non-Newtonian fluid. Applied Mech. and Engg.5(2), 315-327.

Rudraiah, N. (1998). Anatomy and Biomechanics of Synovial Joints - Part II Mathematical Modelling. Recent trends in Basic and Applied anatomy 37105, edited by I. M. Thomas, K. Srinivasa, Rajeshwari, SayeeRajangam, published by Rajeev Gandhi University of Health Science, Karnataka.

Rudraiah, N., S. Kantha and M.N. Manonmani (1998). Anatomy and Biomechanics of Synovial Joints: Part I. Recent Trends in Basic and Applied Anatomy as in Reference above, 1-9.

Rudraiah, N., S.R. Kasiviswanath and P.N. Kaloni (1991). Generalized dispersion in a synovial fluid of human joints. Biorheology 28, 207-219.

Sharma and K.C. Sharma (1978). Rayleigh-Taylor instability of two superposed conducting fluids in presence of suspended particles. Acta. Physica Hungarica 45, 213.

Stokes, V.K. (1968). Effects of Couple Stress in Fluid on Hydromagnetic Channel Flow. Phys. Fluids 11, $1131-1133$.

Takabe, H., K. Mima, L. Montierth and R.L. Morse (1985). Self consistent growth rate of Rayleigh-
Taylor instability in an ablative accelerating plasma. Phy. Fluids 28(12), 3676 
N. Rudraiah and G. Chandrashekara / JAFM, Vol. 3, No. 1, pp. 83-89, 2010.

Table 1 Details of growth rate and reduction

\begin{tabular}{|c|c|c|c|c|c|}
\hline$\sigma$ & $G_{m}$ & $\begin{array}{c}\text { REDUCTION } \\
(\boldsymbol{\%})\end{array}$ & $\sigma$ & $G_{m}$ & $\begin{array}{c}\text { REDUCTION } \\
(\boldsymbol{\%})\end{array}$ \\
\hline 1.05 & 0.023 & 97.6253 & 1.45 & 0.200 & 79.9541 \\
\hline 1.10 & 0.047 & 95.2746 & 1.50 & 0.220 & 77.9685 \\
\hline 1.15 & 0.070 & 92.9548 & 1.55 & 0.239 & 76.0386 \\
\hline 1.20 & 0.093 & 90.6718 & 1.60 & 0.258 & 74.1651 \\
\hline 1.25 & 0.115 & 88.4306 & 1.65 & 0.276 & 72.3479 \\
\hline 1.30 & 0.137 & 86.2353 & 1.70 & 0.294 & 70.5867 \\
\hline 1.35 & 0.159 & 84.0892 & 1.75 & 0.311 & 68.8809 \\
\hline 1.40 & 0.180 & 81.9948 & 1.80 & 0.327 & 67.2299 \\
\hline
\end{tabular}

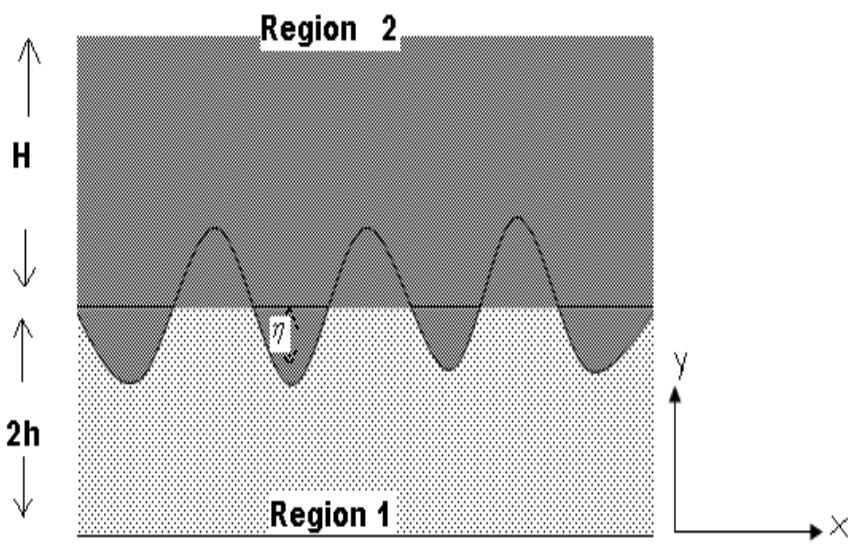

Fig. 1. Physical configuration

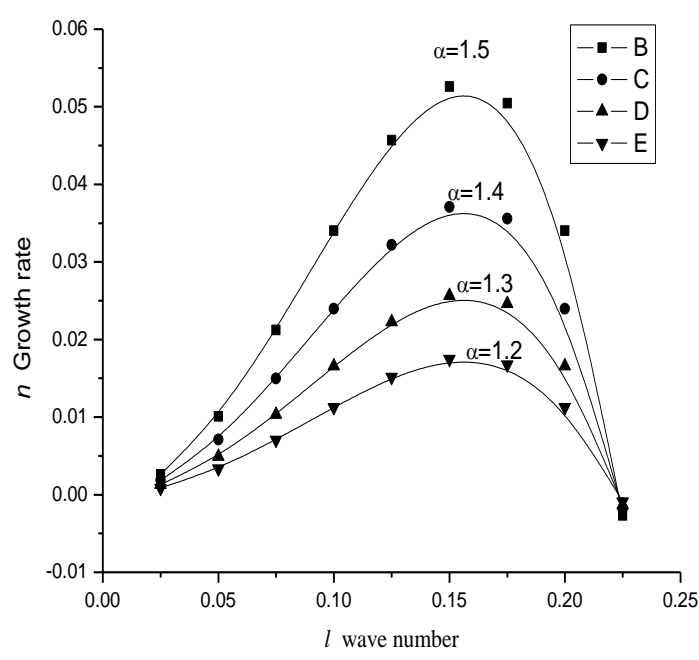

Fig. 2. Graph of dispersion relation versus wave number (varying $\sigma$ )

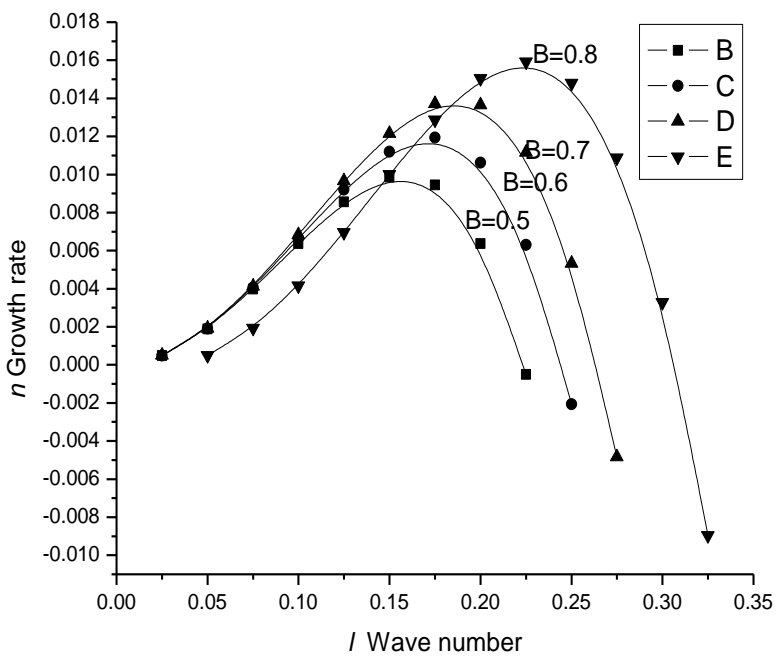

Fig. 3. Graph of dispersion relation versus wave number (varying B) 